\title{
SOME SOLUTIONS FOR FINITELY CONDUCTING VISCOUS MHD PLANE FLOWS*
}

\author{
BY \\ O. P. CHANDNA (University of Windsor) \\ C. E. SOTEROS (Princeton University) \\ M. K. SWAMINATHAN (University of Windsor)
}

1. Introduction. A vast amount of research work has been done to analyse the motion of an electrically conducting fluid moving in a magnetic field since the early theoretical work of Alfven [1]. However, the mathematical complexity of the equations governing these flows has prohibited a thorough analysis thus inducing many researchers to adopt a useful alternative technique of investigating special classes of steady plane or steady axisymmetric flows. This technique requires the imposition of restriction on the angle between the velocity and the magnetic vector fields. Therefore, in the study of steady plane flows in the literature, aligned or parallel, crossed or orthogonal, constantly inclined and transverse flows are dealt with. Any of these restrictions yields a second order mathematical structure conducive to the application of well established fluid-dynamical techniques to the determination of its similarities and its contrasts with the ordinary fluid-dynamics and to obtain solutions. In recent years, the analysis of flow equations for the incompressible MHD flow of an inviscid and viscous fluid having infinite electrical conductivity has been undertaken in various works. Kingston and Talbot [2] studied inviscid orthogonal flows and Waterhouse and Kingston [3] studied constantly inclined flows to classify all possible flows. Chandna and Nath [4] established certain geometrical properties for aligned flows. During the past seven years, Chandna and his coworkers [5-15] have reported a series of results for special classes of plane flow problem, obtaining flow properties, geometries and solutions in the aligned, orthogonal and constantly inclined cases.

In the present paper, the steady viscous incompressible plane flow problem of an electrically conducting fluid having finite electrical conductivity and in the presence of a magnetic field is dealt with. To keep continuity with the previous works in literature, we deal with variably inclined flows. By a variably inclined steady plane flow we mean a flow in which the velocity and the magnetic vector fields are coplanar; the angle between these vector fields is variable and all the flow variables are functions of two space coordinates. Since the magnetic Prandtl number is very small for most liquid metals and it is also small, or at the most of the order of unity, for usual electrically conducting fluids to which MHD (single fluid model) approximation can be applied, our accounting for finite electrical conductivity also makes the flow problem very attractive from both a mathematical and a physical point of view. 
We study the problem with the objective of obtaining exact solutions. Transformations are employed to interchange the roles of the two independent variables and the two components of the magnetic field in the physical plane. These transformations, we shall call the magnetograph transformations, are similar to the well-known hodograph transformations. An excellent survey of the hodograph transformations, one of the strong analytical methods to find solutions in continuum mechanics, has been given by Ames [16]. In the study of MHD, this method has been previously applied to aligned compressible flows by Smith [17], to orthogonal incompressible flows by Chandna and Garg [12] and to constantly inclined flows by Barron and Chandna [14].

The plan of this paper is as follows: in Sec. 2 the basic flow equations are formulated into a convenient form for this work. Sec. 3 deals with the transformation of equations to the magnetograph plane. Under these transformations, it is determined that these flows are governed by a system of two partial differential equations in the Legendre transform function of the magnetic flux function and the transformed variable angle between the vector fields. Theoretical development of Sec. 3 is illustrated by a few rotationally symmetric solutions in Sec. 4.

2. Equations of Motion. The steady, plane flow of a viscous, incompressible fluid of finite electrical conductivity is governed by the following system of equations [18]:

$$
\begin{gathered}
\frac{\partial u}{\partial x}+\frac{\partial v}{\partial y}=0 \\
\rho\left(u \frac{\partial u}{\partial x}+v \frac{\partial u}{\partial y}\right)+\frac{\partial p}{\partial x}=\eta\left(\frac{\partial^{2} u}{\partial x^{2}}+\frac{\partial^{2} u}{\partial y^{2}}\right)-\mu H_{2}\left(\frac{\partial H_{2}}{\partial x}-\frac{\partial H_{1}}{\partial y}\right) \\
\rho\left(u \frac{\partial v}{\partial x}+v \frac{\partial v}{\partial y}\right)+\frac{\partial p}{\partial y}=\eta\left(\frac{\partial^{2} v}{\partial x^{2}}+\frac{\partial^{2} v}{\partial y^{2}}\right)+\mu H_{1}\left(\frac{\partial H_{2}}{\partial x}-\frac{\partial H_{1}}{\partial y}\right) \\
u H_{2}-v H_{1}=\frac{1}{\mu \sigma}\left(\frac{\partial H_{2}}{\partial x}-\frac{\partial H_{1}}{\partial y}\right)+c \\
\frac{\partial H_{1}}{\partial x}+\frac{\partial H_{2}}{\partial y}=0
\end{gathered}
$$

where $u, v$ are the components of the velocity field $\vec{V}, H_{1}, H_{2}$ the components of the magnetic vector field $\vec{H}$, and $p$ is the pressure function; all being functions of $x, y$. In this system $\rho, \eta, \mu, \sigma$, are respectively the constant fluid density, the constant coefficient of viscosity, the constant magnetic permeability, the constant conductivity. Furthermore $c$ is an arbitrary constant of integration obtained from the diffusion equation

$$
\operatorname{curl}\left[[\vec{V} \times \vec{H}]-\frac{1}{\mu \sigma} \operatorname{curl} \vec{H}\right]=0 \text {. }
$$

Introducing the functions

$$
w=\frac{\partial v}{\partial x}-\frac{\partial u}{\partial y}, \quad j=\frac{\partial H_{2}}{\partial x}-\frac{\partial H_{1}}{\partial y}, \quad h=\frac{1}{2} \rho q^{2}+p
$$


where $q^{2}=u^{2}+v^{2}$, the above system of equations is replaced by the following system:

$$
\begin{array}{rlll}
\frac{\partial u}{\partial x}+\frac{\partial v}{\partial y} & = & 0 & \text { (continuity), } \\
\eta \frac{\partial w}{\partial y}-\rho v w+\mu j H_{2} & = & -\frac{\partial h}{\partial x} & \\
\eta \frac{\partial w}{\partial x}-\rho u w+\mu j H_{1} & = & \frac{\partial h}{\partial y} & \\
u H_{2}-v H_{1} & = & \frac{1}{\mu \sigma} j+c & \text { (dinear momentum), } \\
\frac{\partial H_{1}}{\partial x}+\frac{\partial H_{2}}{\partial y} & = & 0 & \\
\frac{\partial v}{\partial x}-\frac{\partial u}{\partial y} & = & w & \text { (solenoidal), } \\
\frac{\partial H_{2}}{\partial x}-\frac{\partial H_{1}}{\partial y} & = & j & \text { (vorticity), } \\
\text { (current density), }
\end{array}
$$

of seven partial differential equations in seven unknowns $u, v, H_{1}, H_{2}, w, j$ and $h$, each functions of $x, y$. This system is advantageously a system of first order. Martin [19] has, with much success, used a similar reduction of order to study viscous non-MHD flows.

We now consider variably inclined plane flows and let $\alpha=\alpha(x, y)$, be the variable angle such that $\alpha(x, y) \neq 0$ for every $(x, y)$ in the flow region. The vector and scalar products of $\vec{V}$ and $\vec{H}$, using the diffusion equation in (2) yield

$$
\begin{aligned}
& u H_{2}-v H_{1}=q H \sin \alpha=\left(c+\frac{1}{\mu \sigma} j\right) \\
& u H_{1}+v H_{2}=q H \cos \alpha=\left(c+\frac{1}{\mu \sigma} j\right) \cos \alpha
\end{aligned}
$$

where $H=\sqrt{H_{1}^{2}+H_{2}^{2}}$. Considering these as two linear algebraic equations in the unknowns $u, v$, we solve (3) in terms of $H_{1}, H_{2}$ and $\alpha$; i.e.

$$
\begin{aligned}
& u=\left(c+\frac{1}{\mu \sigma} j\right)\left[\frac{H_{2}+H_{1} \cot \alpha}{H_{1}^{2}+H_{2}^{2}}\right], \\
& v=\left(c+\frac{1}{\mu \sigma} j\right)\left[\frac{H_{2} \cot \alpha-H_{1}}{H_{1}^{2}+H_{2}^{2}}\right] .
\end{aligned}
$$

Thus one can eliminate $u$ and $v$ from the system (2) by using equation (4). One then obtains a system of equations to be solved for $H_{1}, H_{2}, h, w, j$ and $\alpha$ as functions of $x, y$. This approach leads to the study of system (2) in the magnetograph plane.

Eliminating $u$ and $v$ from the system of equations (2), by using Eq. (4), we obtain the following system of six partial differential equations: 


$$
\begin{aligned}
& \left(c+\frac{1}{\mu \sigma} j\right)\left[H_{1} \frac{\partial \cot \alpha}{\partial x}+H_{2} \frac{\partial \cot \alpha}{\partial y}+\left(\frac{\partial H_{2}}{\partial x}+\frac{\partial H_{1}}{\partial y}\right)\left(\frac{H_{1}^{2}-H_{2}^{2}-2 H_{1} H_{2} \cot \alpha}{H^{2}}\right)\right. \\
& \left.+\left(\frac{\partial H_{2}}{\partial y}-\frac{\partial H_{1}}{\partial x}\right)\left(\frac{H_{1}^{2} \cot \alpha-H_{2}^{2} \cot \alpha+2 H_{1} H_{2}}{H^{2}}\right)\right] \\
& +\frac{1}{\mu \sigma}\left[\frac{\partial j}{\partial x}\left(H_{2}+H_{1} \cot \alpha\right)+\frac{\partial j}{\partial y}\left(H_{2} \cot \alpha-H_{1}\right)\right]=0, \\
& \eta \frac{\partial w}{\partial y}-\rho\left(c+\frac{1}{\mu \sigma} j\right)\left(\frac{H_{2} \cot \alpha-H_{1}}{H^{2}}\right) w+\mu j H_{2}=-\frac{\partial h}{\partial x}, \\
& \eta \frac{\partial w}{\partial x}-\rho\left(c+\frac{1}{\mu \sigma} j\right)\left(\frac{H_{2}+H_{1} \cot \alpha}{H^{2}}\right) w+\mu j H_{1}=\frac{\partial h}{\partial y} \\
& \frac{\partial H_{1}}{\partial x}+\frac{\partial H_{2}}{\partial y}=0 \\
& \frac{\partial H_{2}}{\partial x}-\frac{\partial H_{1}}{\partial y}=j, \\
& \frac{\partial}{\partial x}\left\{\left(c+\frac{1}{\mu \sigma} j\right)\left(\frac{H_{2} \cot \alpha-H_{1}}{H^{2}}\right)\right\}-\frac{\partial}{\partial y}\left\{\left(c+\frac{1}{\mu \sigma} j\right)\left(\frac{H_{2}+H_{1} \cot \alpha}{H^{2}}\right)\right\}=w
\end{aligned}
$$

for six unknown functions $H_{1}, H_{2}, j, w, h, \alpha$ of $x, y$. Once a solution of this equation is determined, the pressure and the velocity functions are obtained by using the definition of $h$ in (1) and equations (4) respectively.

3. Study of flows in the magnetograph plane. Letting the functions $H_{1}=H_{1}(x, y)$, $H_{2}=H_{2}(x, y)$ be such that, in the region of flow, the Jacobian

$$
J(x, y)=\frac{\partial H_{1}}{\partial x} \frac{\partial H_{2}}{\partial y}-\frac{\partial H_{1}}{\partial y} \frac{\partial H_{2}}{\partial x} \neq 0, \quad|J|<\infty,
$$

we may consider $x$ and $y$ as functions of $H_{1}$ and $H_{2}$. By means of $x=x\left(H_{1}, H_{2}\right)$, $y=y\left(H_{1}, H_{2}\right)$, we have the relations

$$
\begin{array}{ll}
\frac{\partial H_{1}}{\partial x}=J \frac{\partial y}{\partial H_{2}}, & \frac{\partial H_{1}}{\partial y}=-J \frac{\partial x}{\partial H_{2}}, \\
\frac{\partial H_{2}}{\partial x}=-J \frac{\partial y}{\partial H_{1}}, & \frac{\partial H_{2}}{\partial y}=J \frac{\partial x}{\partial H_{1}} .
\end{array}
$$

Furthermore, using (7), we have

$$
J(x, y)=\frac{\partial\left(H_{1}, H_{2}\right)}{\partial(x, y)}=\left(\frac{\partial(x, y)}{\partial\left(H_{1}, H_{2}\right)}\right)^{-1}=\bar{J}\left(H_{1}, H_{2}\right)
$$

and

$$
\begin{aligned}
& \frac{\partial f}{\partial x}=\frac{\partial(f, y)}{\partial(x, y)}=J \frac{\partial(f, y)}{\partial\left(H_{1}, H_{2}\right)}=\bar{J} \frac{\partial(\bar{f}, y)}{\partial\left(H_{1}, H_{2}\right)}, \\
& \frac{\partial f}{\partial y}=-\frac{\partial(f, x)}{\partial(x, y)}=J \frac{\partial(x, f)}{\partial\left(H_{1}, H_{2}\right)}=\bar{J} \frac{\partial(x, \bar{f})}{\partial\left(H_{1}, H_{2}\right)}
\end{aligned}
$$


where $f=f(x, y)$ is any continuously differentiable function and $\bar{f}\left(H_{1}, H_{2}\right)$ is its transformed function in the $\left(H_{1}, H_{2}\right)$-plane.

Employing the transformation relations (7) for the first order partial derivatives and Eq. (8) in the system of equations (5), the transformed system of partial differential equations in the $\left(H_{1}, H_{2}\right)$-plane is:

$$
\begin{aligned}
& \frac{\partial x}{\partial H_{1}}+\frac{\partial y}{\partial H_{2}}=0 \\
& \eta \bar{J} \frac{\partial(x, \bar{w})}{\partial\left(H_{1}, H_{2}\right)}-\rho\left(c+\frac{1}{\mu \sigma} \bar{j}\right)\left(\frac{H_{2} \cot \bar{\alpha}-H_{1}}{H^{2}}\right) \bar{w}+\mu \bar{j} H_{2}=-\bar{J} \frac{\partial(\bar{h}, y)}{\partial\left(H_{1}, H_{2}\right)}, \\
& \eta \bar{J} \frac{\partial(\bar{w}, y)}{\partial\left(H_{1}, H_{2}\right)}-\rho\left(c+\frac{1}{\mu \sigma} \bar{j}\right)\left(\frac{H_{2}+H_{1} \cot \bar{\alpha}}{H^{2}}\right) \bar{w}+\mu \bar{j} H_{1}=\bar{J} \frac{\partial(x, \bar{h})}{\partial\left(H_{1}, H_{2}\right)}, \\
& \frac{\partial x}{\partial H_{1}}\left\{\left(c+\frac{1}{\mu \sigma} \bar{j}\right)\left(H_{1}^{2} \cot \bar{\alpha}-H_{2}^{2} \cot \bar{\alpha}+2 H_{1} H_{2}+H_{2} H^{2} \frac{\partial \cot \bar{\alpha}}{\partial H_{2}}\right)\right. \\
& \left.+\frac{H^{2}}{\mu \sigma} \frac{\partial \bar{j}}{\partial H_{2}}\left(H_{2} \cot \bar{\alpha}-H_{1}\right)\right\} \\
& +\frac{\partial x}{\partial H_{2}}\left\{\left(c+\frac{1}{\mu \sigma} \bar{j}\right)\left(H_{2}^{2}-H_{1}^{2}+2 H_{1} H_{2} \cot \bar{\alpha}-H_{2} H^{2} \frac{\partial \cot \bar{\alpha}}{\partial H_{1}}\right)\right. \\
& \left.+\frac{H^{2}}{\mu \sigma} \frac{\partial \bar{j}}{\partial H_{1}}\left(H_{1}-H_{2} \cot \bar{\alpha}\right)\right\} \\
& +\frac{\partial y}{\partial H_{2}}\left\{\left(c+\frac{1}{\mu \sigma} \bar{j}\right)\left(H_{2}^{2} \cot \bar{\alpha}-H_{1}^{2} \cot \bar{\alpha}-2 H_{1} H_{2}+H_{1} H^{2} \frac{\partial \cot \bar{\alpha}}{\partial H_{1}}\right)\right. \\
& \left.+\frac{H^{2}}{\mu \sigma} \frac{\partial \bar{j}}{\partial H_{1}}\left(H_{1} \cot \bar{\alpha}+H_{2}\right)\right\} \\
& +\frac{\partial y}{\partial H_{1}}\left\{\left(c+\frac{1}{\mu \sigma} \bar{j}\right)\left(H_{2}^{2}-H_{1}^{2}+2 H_{1} H_{2} \cot \bar{\alpha}-H_{1} H^{2} \frac{\partial \cot \bar{\alpha}}{\partial H_{2}}\right)\right. \\
& \left.-\frac{H^{2}}{\mu \sigma} \frac{\partial \bar{j}}{\partial H_{2}}\left(H_{1} \cot \bar{\alpha}+H_{2}\right)\right\}=0 \text {, } \\
& \bar{J}\left(\frac{\partial x}{\partial H_{2}}-\frac{\partial y}{\partial H_{1}}\right)=\bar{j}, \\
& \bar{J}\left[\frac{\partial\left(\left(c+\frac{1}{\mu \sigma} \bar{j}\right)\left(\frac{H_{2} \cot \bar{\alpha}-H_{1}}{H^{2}}\right), y\right)}{\partial\left(H_{1}, H_{2}\right)}\right. \\
& \left.-\frac{\partial\left(x,\left(c+\frac{1}{\mu \sigma} \bar{j}\right)\left(\frac{H_{2}+H_{1} \cot \bar{\alpha}}{H^{2}}\right)\right)}{\partial\left(H_{1}, H_{2}\right)}\right]=\bar{w} .
\end{aligned}
$$


This is a system of six partial differential equations in six unknown functions $x\left(H_{1}, H_{2}\right)$, $y\left(H_{1}, H_{2}\right)$ and the four transformed functions $\bar{w}\left(H_{1}, H_{2}\right), \bar{h}\left(H_{1}, H_{2}\right), \bar{j}\left(H_{1}, H_{2}\right), \bar{\alpha}\left(H_{1}, H_{2}\right)$ when $\bar{J}=\left(\partial(x, y) / \partial\left(H_{1}, H_{2}\right)\right)^{-1}$ is employed from (8). Once a solution set $x=x\left(H_{1}, H_{2}\right)$, $y=y\left(H_{1}, H_{2}\right), \bar{w}=\bar{w}\left(H_{1}, H_{2}\right), \bar{h}=\bar{h}\left(H_{1}, H_{2}\right), \bar{j}=\bar{j}\left(H_{1}, H_{2}\right), \bar{\alpha}=\bar{\alpha}\left(H_{1}, H_{2}\right)$ is determined for this system, we are lead to the solutions $H_{1}=H_{1}(x, y), H_{2}=H_{2}(x, y)$ and therefore $w=w(x, y), h=h(x, y), j=j(x, y), \alpha=\alpha(x, y)$ for the previous system of equations (5).

The solenoidal equation implies the existence of a magnetic flux function $\phi(x, y)$ such that

$$
d \phi=-H_{2} d x+H_{1} d y \quad \text { or } \quad \frac{\partial \phi}{\partial x}=-H_{2}, \frac{\partial \phi}{\partial y}=H_{1} .
$$

Likewise, Eq. (10) implies the existence of a function $L\left(H_{1}, H_{2}\right)$, called the Legendre transform function of the magnetic flux function $\phi(x, y)$, so that

$$
d L=-y d H_{1}+x d H_{2} \quad \text { or } \quad \frac{\partial L}{\partial H_{1}}=-y, \frac{\partial L}{\partial H_{2}}=x
$$

and the two functions $\phi(x, y), L\left(H_{1}, H_{2}\right)$ are related by

$$
L\left(H_{1}, H_{2}\right)=H_{2} x-H_{1} y+\phi(x, y) .
$$

Introducing $L\left(H_{1}, H_{2}\right)$ into the system (10) to (15), with $\bar{J}$ given by (8), it follows that equation (10) is identically satisfied and this system may be replaced by

$$
\begin{gathered}
\eta \bar{J} \frac{\partial\left(\partial L / \partial H_{2}, \bar{w}\right)}{\partial\left(H_{1}, H_{2}\right)}-\rho\left(c+\frac{1}{\mu \sigma} \bar{j}\right)\left(\frac{H_{2} \cot \bar{\alpha}-H_{1}}{H^{2}}\right) \bar{w}+\mu \bar{j} H_{2}=\bar{J} \frac{\partial\left(\bar{h}, \partial L / \partial H_{1}\right)}{\partial\left(H_{1}, H_{2}\right)}, \\
\eta \bar{J} \frac{\partial\left(\bar{w}, \partial L / \partial H_{1}\right)}{\partial\left(H_{1}, H_{2}\right)}+\rho\left(c+\frac{1}{\mu \sigma} \bar{j}\right)\left(\frac{H_{2}+H_{1} \cot \bar{\alpha}}{H^{2}}\right) \bar{w}-\mu \bar{j} H_{1}=-\bar{J} \frac{\partial\left(\partial L / \partial H_{2}, \bar{h}\right)}{\partial\left(H_{1}, H_{2}\right)},
\end{gathered}
$$

$$
\begin{gathered}
\frac{\partial^{2} L}{\partial H_{2}^{2}}\left\{\left(c+\frac{1}{\mu \sigma} \bar{j}\right)\left(H_{2}^{2}-H_{1}^{2}+2 H_{1} H_{2} \cot \bar{\alpha}-H_{2} H^{2} \frac{\partial \cot \bar{\alpha}}{\partial H_{1}}\right)\right. \\
\left.+\frac{H^{2}}{\mu \sigma}\left(H_{1}-H_{2} \cot \bar{\alpha}\right) \frac{\partial \bar{j}}{\partial H_{1}}\right\} \\
+\frac{\partial^{2} L}{\partial H_{1} \partial H_{2}}\left\{\left(c+\frac{1}{\mu \sigma} \bar{j}\right) H^{2}\left(H_{2} \frac{\partial \cot \bar{\alpha}}{\partial H_{2}}-H_{1} \frac{\partial \cot \bar{\alpha}}{\partial H_{1}}\right)\right. \\
+\frac{H^{2}}{\mu \sigma}\left(\left(H_{2} \cot \bar{\alpha}-H_{1}\right) \frac{\partial \bar{j}}{\partial H_{2}}-\left(H_{1} \cot \bar{\alpha}+H_{2}\right) \frac{\partial \bar{j}}{\partial H_{1}}\right) \\
\left.+\left(c+\frac{1}{\mu \sigma} \bar{j}\right)\left(2 H_{1}^{2} \cot \bar{\alpha}-2 H_{2}^{2} \cot \bar{\alpha}+4 H_{1} H_{2}\right)\right\}
\end{gathered}
$$




$$
\begin{aligned}
& -\frac{\partial^{2} L}{\partial H_{1}^{2}}\left\{( c + \frac { 1 } { \mu \sigma } \overline { j } ) \left(H_{2}^{2}-H_{1}^{2}+2 H_{1} H_{2} \cot \bar{\alpha}\right.\right. \\
& \left.\left.-H_{1} H^{2} \frac{\partial \cot \bar{\alpha}}{\partial H_{2}}\right)-\frac{H^{2}}{\mu \sigma}\left(H_{1} \cot \bar{\alpha}+H_{2}\right) \frac{\partial \bar{j}}{\partial H_{2}}\right\}=0, \\
& \bar{J}\left(\frac{\partial^{2} L}{\partial H_{2}^{2}}+\frac{\partial^{2} L}{\partial H_{1}^{2}}\right)=\bar{j} \\
& \partial\left(\left(c+\frac{1}{\mu \sigma} \bar{j}\right)\left(\frac{H_{2} \cot \bar{\alpha}-H_{1}}{H^{2}}\right), \frac{\partial L}{\partial H_{1}}\right) / \partial\left(H_{1}, H_{2}\right) \\
& +\partial\left(\frac{\partial L}{\partial H_{2}},\left(c+\frac{1}{\mu \sigma} \bar{j}\right)\left(\frac{H_{2}+H_{1} \cot \bar{\alpha}}{H^{2}}\right)\right) / \partial\left(H_{1}, H_{2}\right)=-\bar{w} / \bar{J}
\end{aligned}
$$

with

$$
\bar{J}=\left[\frac{\partial^{2} L}{\partial H_{1}^{2}} \frac{\partial^{2} L}{\partial H_{2}^{2}}-\left(\frac{\partial^{2} L}{\partial H_{1} \partial H_{2}}\right)^{2}\right]^{-1}
$$

for the six functions $L\left(H_{1}, H_{2}\right), \bar{h}\left(H_{1}, H_{2}\right), \bar{w}\left(H_{1}, H_{2}\right), \bar{j}\left(H_{1}, H_{2}\right), \bar{\alpha}\left(H_{1}, H_{2}\right)$ and $\bar{J}\left(H_{1}, H_{2}\right)$.

We now define

$$
\begin{aligned}
& Q_{1}\left(H_{1}, H_{2}\right)=\frac{\partial\left(\partial L / \partial H_{2}, \bar{w}\right)}{\partial\left(H_{1}, H_{2}\right)}, \\
& Q_{2}\left(H_{1}, H_{2}\right)=\frac{\partial\left(\partial L / \partial H_{1}, \bar{w}\right)}{\partial\left(H_{1}, H_{2}\right)}
\end{aligned}
$$

and use the integrability condition

$$
\begin{aligned}
\left(\bar{J} \frac{\partial^{2} L}{\partial H_{1} \partial H_{2}} \frac{\partial}{\partial H_{2}}-\right. & \left.\bar{J} \frac{\partial^{2} L}{\partial H_{2}^{2}} \frac{\partial}{\partial H_{1}}\right)\left[\bar{J} \frac{\partial\left(\partial L / \partial H_{1}, \bar{h}\right)}{\partial\left(H_{1}, H_{2}\right)}\right] \\
& =\left(\bar{J} \frac{\partial^{2} L}{\partial H_{1}^{2}} \frac{\partial}{\partial H_{2}}-\bar{J} \frac{\partial^{2} L}{\partial H_{1} \partial H_{2}} \frac{\partial}{\partial H_{1}}\right)\left[\bar{J} \frac{\partial\left(\partial L / \partial H_{2}, \bar{h}\right)}{\partial\left(H_{1}, H_{2}\right)}\right],
\end{aligned}
$$

i.e. $\partial^{2} h / \partial x \partial y=\partial^{2} h / \partial y \partial x$, along with Eq. (20) to eliminate $\bar{h}\left(H_{1}, H_{2}\right)$ from equations (18) and (19) to obtain

$$
\begin{gathered}
\eta\left(\frac{\partial\left(\partial L / \partial H_{2}, \bar{J} Q_{1}\right)}{\partial\left(H_{1}, H_{2}\right)}+\frac{\partial\left(\partial L / \partial H_{1}, \bar{J} Q_{2}\right)}{\partial\left(H_{1}, H_{2}\right)}\right) \\
+\mu H_{1} \frac{\partial\left(\partial L / \partial H_{1}, \bar{j}\right)}{\partial\left(H_{1}, H_{2}\right)}+\mu H_{2} \frac{\partial\left(\partial L / \partial H_{2}, \bar{j}\right)}{\partial\left(H_{1}, H_{2}\right)} \\
-\frac{\rho}{H^{2}}\left(c+\frac{1}{\mu \sigma} \bar{j}\right)\left(Q_{1}\left(H_{2} \cot \bar{\alpha}-H_{1}\right)+Q_{2}\left(H_{2}+H_{1} \cot \bar{\alpha}\right)\right)=0 .
\end{gathered}
$$


Summing up, we have the following theorem:

THEOREM I. If $L\left(H_{1}, H_{2}\right)$ is the Legendre transform function of a magnetic flux function of the equations governing the plane steady flow of a viscous incompressible fluid of finite electrical conductivity and $\bar{\alpha}\left(H_{1}, H_{2}\right)$ is the angle of the variably inclined plane flows, then $L\left(H_{1}, H_{2}\right)$ and $\bar{\alpha}\left(H_{1}, H_{2}\right)$ must satisfy (20) and (26), where $\bar{j}\left(H_{1}, H_{2}\right), \bar{J}\left(H_{1}, H_{2}\right)$, $Q_{1}\left(H_{1}, H_{2}\right), Q_{2}\left(H_{1}, H_{2}\right)$ are given by Eqs. (2l) to (25).

Once a solution $L=L\left(H_{1}, H_{2}\right), \bar{\alpha}=\bar{\alpha}\left(H_{1}, H_{2}\right)$ of Eqs. (20), (26) is found, for which $\bar{J}$ evaluated from (23) satisfies $0<|J|<\infty$, the solutions for the magnetic field components $H_{1}(x, y), H_{2}(x, y)$ are obtained by solving simultaneously

$$
\frac{\partial L}{\partial H_{1}}=-y, \quad \frac{\partial L}{\partial H_{2}}=x .
$$

Having obtained magnetic field components in the physical plane, we obtain $\alpha$ in the physical plane from the solution for $\bar{\alpha}$ in the magnetograph plane. Finally, $w(x, y)$, $h(x, y), j(x, y), u(x, y), v(x, y)$, and $p(x, y)$ are determined by using system (5) and Eqs. (4), (1).

We now write Eqs. (20), (26) in polar coordinates $(H, \theta)$. We have

$$
H=\sqrt{H_{1}^{2}+H_{2}^{2}}, \quad \theta=\tan ^{-1} \frac{H_{2}}{H_{1}} \quad \text { or } \quad H_{1}=H \cos \theta, \quad H_{2}=H \sin \theta
$$

and

$$
\frac{\partial}{\partial H_{1}}=\cos \theta \frac{\partial}{\partial H}-\frac{\sin \theta}{H} \frac{\partial}{\partial \theta}, \quad \frac{\partial}{\partial H_{2}}=\sin \theta \frac{\partial}{\partial H}+\frac{\cos \theta}{H} \frac{\partial}{\partial \theta} .
$$

Defining $L^{*}(H, \theta), \alpha^{*}(H, \theta), w^{*}(H, \theta), j^{*}(H, \theta), J^{*}(H, \theta)$ to be the Legendre transform, variable angle, vorticity, current density, jacobian function in $(H, \theta)$-coordinates, and using

$$
\frac{\partial(F, G)}{\partial\left(H_{1}, H_{2}\right)}=\frac{\partial\left(F^{*}, G^{*}\right)}{\partial(H, \theta)} \frac{\partial(H, \theta)}{\partial\left(H_{1}, H_{2}\right)}=\frac{1}{H} \frac{\partial\left(F^{*}, G^{*}\right)}{\partial(H, \theta)},
$$

where $F\left(H_{1}, H_{2}\right)=F^{*}(H, \theta), G\left(H_{1}, H_{2}\right)=G^{*}(H, \theta)$ are continuously differentiable functions, we obtain the following corollary to Theorem I.

Corollary. If $L^{*}(H, \theta)$ is the Legendre transform function of a magnetic flux function of the equations governing the plane steady flow of a viscous incompressible fluid of finite electrical conductivity with variable angle $\alpha^{*}(H, \theta)$, then $L^{*}(H, \theta)$ and $\alpha^{*}(H, \theta)$ must satisfy

$$
\begin{aligned}
& \frac{\partial^{2} L^{*}}{\partial H_{2}}\left\{\left(c+\nu_{H} j^{*}\right)\left(1+\frac{\partial \cot \alpha^{*}}{\partial \theta}\right)+\nu_{H} \cot \alpha^{*} \frac{\partial j^{*}}{\partial \theta}\right\} \\
& +\left(\frac{1}{H^{2}} \frac{\partial^{2} L^{*}}{\partial \theta^{2}}+\frac{1}{H} \frac{\partial L^{*}}{\partial H}\right)\left\{H \nu_{H} \frac{\partial j^{*}}{\partial H}-\left(c+\nu_{H} j^{*}\right)\right\} \\
& +\left(\frac{1}{H} \frac{\partial^{2} L^{*}}{\partial \theta \partial H}-\frac{1}{H^{2}} \frac{\partial L^{*}}{\partial \theta}\right)\left\{\left(c+\nu_{H} j^{*}\right)\left(2 \cot \alpha^{*}-H \frac{\partial \cot \alpha^{*}}{\partial H}\right)\right. \\
& \left.-\cot \alpha^{*} H \nu_{H} \frac{\partial j^{*}}{\partial H}-\nu_{H} \frac{\partial j^{*}}{\partial \theta}\right\}=0
\end{aligned}
$$




$$
\begin{aligned}
\eta\left(\partial\left(\sin \theta \frac{\partial L^{*}}{\partial H}+\frac{\cos \theta}{H} \frac{\partial L^{*}}{\partial \theta}, J^{*} Q_{1}^{*}\right) / \partial(H, \theta)\right. \\
\left.\quad+\partial\left(\cos \theta \frac{\partial L^{*}}{\partial H}-\frac{\sin \theta}{H} \frac{\partial L^{*}}{\partial \theta}, J^{*} Q_{2}^{*}\right) / \partial(H, \theta)\right) \\
\quad+\mu H \sin \theta \partial\left(\sin \theta \frac{\partial L^{*}}{\partial H}+\frac{\cos \theta}{H} \frac{\partial L^{*}}{\partial \theta}, j^{*}\right) / \partial(H, \theta) \\
\quad+\mu H \cos \theta \partial\left(\cos \theta \frac{\partial L^{*}}{\partial H}-\frac{\sin \theta}{H} \frac{\partial L^{*}}{\partial \theta}, j^{*}\right) / \partial(H, \theta) \\
\quad-\rho\left(c+\nu_{H} j^{*}\right)\left(Q_{1}^{*}\left(\sin \theta \cot \alpha^{*}-\cos \theta\right)+Q_{2}^{*}\left(\sin \theta+\cos \theta \cot \alpha^{*}\right)\right)=0
\end{aligned}
$$

where $\nu_{H}=1 / \mu \sigma$ is the magnetic viscosity and $J^{*}, Q_{1}^{*}, Q_{2}^{*}, j^{*}, w^{*}$ are given by

$$
\begin{aligned}
& J^{*}(H, \theta)=H^{4} /\left[H^{2} \frac{\partial^{2} L^{*}}{\partial H^{2}}\left(H \frac{\partial L^{*}}{\partial H}+\frac{\partial^{2} L^{*}}{\partial \theta^{2}}\right)-\left(\frac{\partial L^{*}}{\partial \theta}-H \frac{\partial^{2} L^{*}}{\partial H \partial \theta}\right)^{2}\right] \\
& j^{*}(H, \theta)=J^{*}\left(\frac{\partial^{2} L^{*}}{\partial H^{2}}+\frac{1}{H^{2}} \frac{\partial^{2} L^{*}}{\partial \theta^{2}}+\frac{1}{H} \frac{\partial L^{*}}{\partial H}\right) \\
& Q_{1}^{*}(H, \theta)=\frac{1}{H}\left[\partial\left(\sin \theta \frac{\partial L^{*}}{\partial H}+\frac{\cos \theta}{H} \frac{\partial L^{*}}{\partial \theta}, w^{*}\right) / \partial(H, \theta)\right] \\
& Q_{2}^{*}(H, \theta)=\frac{1}{H}\left[\partial\left(\cos \theta \frac{\partial L^{*}}{\partial H}-\frac{\sin \theta}{H} \frac{\partial L^{*}}{\partial \theta}, w^{*}\right) / \partial(H, \theta)\right]
\end{aligned}
$$

and

$$
\begin{aligned}
w^{*}(H, \theta)= & \frac{J^{*}}{H^{2}}\left\{( c + \nu _ { H } j ^ { * } ) \left[\frac{\partial \cot \alpha^{*}}{\partial \theta}\left(\frac{1}{H^{2}} \frac{\partial L^{*}}{\partial \theta}-\frac{1}{H} \frac{\partial^{2} L^{*}}{\partial \theta \partial H}\right)\right.\right. \\
& +\frac{\partial \cot \alpha}{\partial H}\left(\frac{\partial L^{*}}{\partial H}+\frac{1}{H} \frac{\partial^{2} L^{*}}{\partial \theta^{2}}\right) \\
& +\cot \alpha^{*}\left(\frac{\partial^{2} L^{*}}{\partial H^{2}}-\frac{1}{H} \frac{\partial L^{*}}{\partial H}-\frac{1}{H^{2}} \frac{\partial^{2} L^{*}}{\partial \theta^{2}}\right) \\
& \left.+\frac{2}{H}\left(\frac{1}{H} \frac{\partial L^{*}}{\partial \theta}-\frac{\partial^{2} L^{*}}{\partial \theta \partial H}\right)\right]-\nu_{H}\left[\frac{\cot \alpha^{*}}{H} \frac{\partial j^{*}}{\partial \theta}\left(\frac{\partial^{2} L^{*}}{\partial \theta \partial H}-\frac{1}{H} \frac{\partial L^{*}}{\partial \theta}\right)\right. \\
& -\cot \alpha^{*} \frac{\partial j^{*}}{\partial H}\left(\frac{\partial L^{*}}{\partial H}+\frac{1}{H} \frac{\partial^{2} L^{*}}{\partial \theta^{2}}\right) \\
& \left.\left.+\frac{\partial j^{*}}{\partial \theta} \frac{\partial^{2} L^{*}}{\partial H^{2}}+\frac{\partial j^{*}}{\partial H}\left(\frac{1}{H} \frac{\partial L^{*}}{\partial \theta}-\frac{\partial^{2} L}{\partial \theta \partial H}\right)\right]\right\} .
\end{aligned}
$$

Once a solution $L^{*}=L^{*}(H, \theta), \alpha^{*}=\alpha^{*}(H, \theta)$ of the system of Eqs. (30), (31) is determined, we employ

$$
\begin{aligned}
& x=\sin \theta \frac{\partial L^{*}}{\partial H}+\frac{\cos \theta}{H} \frac{\partial L^{*}}{\partial \theta}, \\
& y=\frac{\sin \theta}{H} \frac{\partial L^{*}}{\partial \theta}-\cos \theta \frac{\partial L^{*}}{\partial H}
\end{aligned}
$$


and (28) to obtain $H_{1}=H_{1}(x, y), H_{2}=H_{2}(x, y)$ in the physical plane. The remaining flow variables are then obtained, in the physical plane, by using the flow equations in the physical plane.

4. Applications. In this section we investigate various flow problems as applications of Theorem I and its corollary.

Application 1. Let

$$
L^{*}(H, \theta)=F(H)
$$

be the Legendre transform function of the magnetic flux function in $(H, \theta)$-coordinates such that $F^{\prime}(H) \neq 0, F^{\prime \prime}(H) \neq 0$.

Using (38) in (30), we find that $\alpha^{*}(H, \theta)$ satisfies

$$
\frac{\partial \cot \alpha^{*}}{\partial \theta}=\frac{1}{\left(c+\nu_{H} j^{*}\right)}\left[c\left(\frac{F^{\prime}}{H F^{\prime \prime}}-1\right)+\nu_{H}\left(\frac{F^{\prime}}{H\left(F^{\prime \prime}\right)^{2}}-\frac{1}{F^{\prime \prime}}+\frac{F^{\prime \prime \prime} F^{\prime}}{\left(F^{\prime \prime}\right)^{3}}\right)\right]
$$

where

$$
j^{*}=\frac{H}{F^{\prime}}+\frac{1}{F^{\prime \prime}}
$$

as calculated from Eq. (33). Therefore its integration yields

$$
\cot \alpha^{*}=G_{1}(H) \theta+G_{2}(H)
$$

where

$$
G_{1}(H)=\frac{1}{\left(c+\nu_{H} j^{*}\right)}\left[c\left(\frac{F^{\prime}}{H F^{\prime \prime}}-1\right)+\nu_{H}\left(\frac{F^{\prime}}{H\left(F^{\prime \prime}\right)^{2}}-\frac{1}{F^{\prime \prime}}+\frac{F^{\prime \prime \prime} F^{\prime}}{\left(F^{\prime \prime}\right)^{3}}\right)\right]
$$

and $G_{2}(H)$ is an arbitrary function of $H$.

By using (38) and (41) in (32) to (36), the expressions for $w^{*}, Q_{1}^{*}, Q_{2}^{*}, J^{*}$ are found to be

$$
\begin{aligned}
& w^{*}=A(H) \theta+B(H) \\
& Q_{1}^{*}=\frac{F^{\prime \prime}}{H} A(H) \sin \theta-\left(\frac{F^{\prime}}{H} \cos \theta\right)\left(A^{\prime}(H) \theta+B^{\prime}(H)\right) \\
& Q_{2}^{*}=\frac{F^{\prime \prime}}{H} A(H) \cos \theta+\left(\frac{F^{\prime}}{H} \sin \theta\right)\left(A^{\prime}(H) \theta+B^{\prime}(H)\right) \\
& J^{*}=\frac{H}{F^{\prime \prime} F^{\prime}}
\end{aligned}
$$


where

$$
\begin{aligned}
A(H)= & \frac{c G_{1}^{\prime}}{H F^{\prime \prime}}+c G_{1}\left(\frac{1}{H F^{\prime}}-\frac{1}{H^{2} F^{\prime \prime}}\right)+\nu_{H} G_{1}^{\prime}\left(\frac{1}{F^{\prime \prime} F^{\prime}}+\frac{1}{H\left(F^{\prime \prime}\right)^{2}}\right) \\
& +\nu_{H} G_{1}\left(\frac{1}{H F^{\prime \prime} F^{\prime}}-\frac{1}{H^{2}\left(F^{\prime \prime}\right)^{2}}-\frac{F^{\prime \prime}}{H\left(F^{\prime \prime}\right)^{3}}\right) \\
B(H)= & \frac{c G_{2}^{\prime}}{H F^{\prime \prime}}+c G_{2}\left(\frac{1}{H F^{\prime}}-\frac{1}{H^{2} F^{\prime \prime}}\right)+\nu_{H} G_{2}^{\prime}\left(\frac{1}{F^{\prime \prime} F^{\prime}}+\frac{1}{H\left(F^{\prime \prime}\right)^{2}}\right) \\
& +\nu_{H} G_{2}\left(\frac{1}{H F^{\prime \prime} F^{\prime}}-\frac{1}{H^{2}\left(F^{\prime \prime}\right)^{2}}-\frac{F^{\prime \prime \prime}}{H\left(F^{\prime \prime}\right)^{3}}\right)
\end{aligned}
$$

Thus $L^{*}(H, \theta)$ and $\cot \alpha^{*}$, given by (38) and (41) respectively, satisfy equation (30) but, in order to be a solution set of equations (30), (31), the unknown functions $F(H)$ and $G_{2}(H)$ must satisfy

$$
\begin{aligned}
\frac{\rho}{H}\left(c+\nu_{H} j^{*}\right)\left(\cot \alpha^{*} \frac{\partial w^{*}}{\partial \theta} F^{\prime \prime}+\frac{\partial w^{*}}{\partial H} F^{\prime}\right) \\
-\eta\left(\frac{\partial\left(\sin \theta F^{\prime}, J^{*} Q_{1}^{*}\right)}{\partial(H, \theta)}+\frac{\partial\left(\cos \theta F^{\prime}, J^{*} Q_{2}^{*}\right)}{\partial(H, \theta)}\right)=0
\end{aligned}
$$

with $j^{*}, \cot \alpha^{*}, w^{*}, Q_{1}^{*}, Q_{2}^{*}, J^{*}$ as in (40) to (43).

We find that

$$
\begin{aligned}
F(H) & =M_{1} H^{2}+M_{2}, \\
G_{2}(H) & =M_{3} H^{2}+M_{4}
\end{aligned}
$$

is a solution set of (45) such that $F^{\prime}(H) \neq 0, F^{\prime \prime}(H) \neq 0$, where $M_{1} \neq 0, M_{2}, M_{3}, M_{4}$ are arbitrary constants.

Therefore

$$
\begin{aligned}
& L^{*}(H, \theta)=M_{1} H^{2}+M_{2} \text { i.e. } L\left(H_{1}, H_{2}\right)=M_{1}\left(H_{1}^{2}+H_{2}^{2}\right)=M_{2} \\
& \alpha^{*}(H, \theta)=\cot ^{-1}\left(M_{3} H^{2}+M_{4}\right) \text { i.e. } \alpha\left(H_{1}, H_{2}\right)=\cot ^{-1}\left(M_{3} H_{1}^{2}+M_{3} H_{2}^{2}+M_{4}\right)
\end{aligned}
$$

forms a solution set of the system of partial differential equations (30), (31) or (20), (26).

As the constants $M_{3}, M_{4}$ are arbitrary, three separate cases arise from the solution (47). If $M_{3} \neq 0, M_{4} \neq 0$, then the flows are variably inclined. If $M_{3}=0, M_{4} \neq 0$, the resulting flows are constantly inclined and finally, if $M_{3}=M_{4}=0$, the flow are crossed.

Taking the first case, $L^{*}(H, \theta), \alpha^{*}(H, \theta)$ are given by (47). Using (47) in Eqs. (37), (40) to (43), expressions for the magnetic field components, the vorticity and the current density are obtained as

$$
H_{1}=-\frac{y}{2 M_{1}}, \quad H_{2}=\frac{x}{2 M_{1}}, \quad w=\frac{M_{3}}{M_{1}}\left(c+\frac{\nu_{H}}{M_{1}}\right), \quad j=\frac{1}{M_{1}} .
$$


In the physical plane, the variable angle between the velocity and magnetic fields, is obtained by using (48) in (47) to give

$$
\alpha(x, y)=\cot ^{-1}\left[\frac{M_{3}\left(x^{2}+y^{2}\right)}{4 M_{1}^{2}}+M_{4}\right]
$$

and therefore, the velocity components, by using (48) and (49) in (4), are given by

$$
\begin{aligned}
& u(x, y)=\left(c+\frac{\nu_{H}}{M_{1}}\right)\left(\frac{2 M_{1}\left(x-M_{4} y\right)}{x^{2}+y^{2}}-\frac{M_{3} y}{2 M_{1}}\right), \\
& v(x, y)=\left(c+\frac{\nu_{H}}{M_{1}}\right)\left(\frac{2 M_{1}\left(y+M_{4} x\right)}{x^{2}+y^{2}}+\frac{M_{3} x}{2 M_{1}}\right) .
\end{aligned}
$$

Using (48) to (50) in the linear momentum equations of system (2) and integrating, we find the function $h(x, y)$. Using this solution for $h(x, y)$ and (48) in (1), the pressure function is

$$
\begin{aligned}
p(x, y)=p\left(c+\frac{\nu_{H}}{M_{1}}\right)^{2}\left(2 M_{3} \tan ^{-1}\left(\frac{y}{x}\right)+\frac{M_{3}^{2}}{8 M_{1}^{2}}\left(x^{2}+y^{2}\right)-\frac{2 M_{1}\left(1+M_{4}^{2}\right)}{x^{2}+y^{2}}\right. \\
\left.-M_{3} M_{4}\left(1-\ln \left(x^{2}+y^{2}\right)\right)\right) \\
-\frac{\mu}{4 M_{1}^{2}}\left(x^{2}+y^{2}\right)+M_{5}
\end{aligned}
$$

where $M_{5}$ is an arbitrary constant.

Finally, we find the magnetic lines are $x^{2}+y^{2}=$ const. and the streamlines are $M_{3}\left(x^{2}+y^{2}\right)-8 M_{1}^{2} \tan ^{-1}(y / x)+4 M_{1}^{2} M_{4} \ln \left(x^{2}+y^{2}\right)=$ const.

Summing up the result of this case, we have

THEOREM II. If the flow is a general MHD steady plane flow, then $L^{*}(H, \theta)=M_{1} H^{2}+M_{2}$ is a Legendre transform function of a magnetic flux function such that the streamlines and the magnetic lines corresponding to this Legendre transform function and the variable angle

$$
\alpha(x, y)=\cot ^{-1}\left[\frac{M_{3}\left(x^{2}+y^{2}\right)}{4 M_{1}^{2}}+M_{4}\right]
$$

are

$$
\begin{aligned}
& M_{3}\left(x^{2}+y^{2}\right)-8 M_{1}^{2} \tan ^{-1}\left(\frac{y}{x}\right)-4 M_{1}^{2} M_{4} \ln \left(x^{2}+y^{2}\right)=\text { const., } \\
& x^{2}+y^{2}=\text { const. }
\end{aligned}
$$

The solutions to the other cases can be obtained from (48) to (51) by taking $M_{3}=0$ and $M_{3}=M_{4}=0$ respectively. 
Applications 2. Consider now the Legendre transform function of the magnetic flux function in $(H, \theta)$-coordinates in the form

$$
L^{*}(H, \theta)=F(\theta)
$$

such that $F^{\prime}(\theta) \neq 0$.

From substituting (52) into (30), we have the partial differential equation

$$
\left(\frac{\partial \cot \alpha^{*}}{\partial H}\right) F^{\prime} H\left(c+\nu_{H} j^{*}\right)-2 \cot \alpha^{*} F^{\prime} c-F^{\prime \prime}\left(c+\nu_{H} j^{*}\right)-\frac{H^{2} F^{\prime \prime \prime}}{F^{\prime}} \nu_{H}=0
$$

in the unknown functions $F(\theta), \alpha^{*}(H, \theta)$. Furthermore, (31) becomes

$$
\begin{aligned}
\frac{\rho}{H^{2}}(C+ & \left.\nu_{H} j^{*}\right)\left[\frac{\partial w^{*}}{\partial H}\left(\cot \alpha^{*} F^{\prime}+F^{\prime \prime}\right)+\frac{\partial w^{*}}{\partial \theta} \frac{F^{\prime}}{H}\right]+2 \mu \frac{H F^{\prime \prime}}{F^{\prime}} \\
& -\eta\left(\partial\left(\frac{\cos \theta}{H} F^{\prime}, J^{*} Q_{1}^{*}\right) / \partial(H, \theta)+\partial\left(-\frac{\sin \theta}{H} F^{\prime}, J^{*} Q_{2}^{*}\right) / \partial(H, \theta)\right)=0
\end{aligned}
$$

where

$$
\begin{gathered}
J^{*}(H, \theta)=-\frac{H^{4}}{\left(F^{\prime}\right)^{2}}, \quad j^{*}(H, \theta)=-\frac{H^{2} F^{\prime \prime}}{\left(F^{\prime}\right)^{2}}, \\
w^{*}(H, \theta)=J^{*}\left[\frac{\partial \cot \alpha^{*}}{\partial \theta} \frac{F^{\prime}}{H^{4}}\left(c+\nu_{H} j^{*}\right)+\frac{\partial \cot \alpha^{*}}{\partial H} \frac{F^{\prime \prime}}{H^{3}}\left(c+\nu_{H} j^{*}\right)\right. \\
\left.-\frac{\cot \alpha^{*}}{H^{3}}\left(\frac{F^{\prime \prime} c}{H}-\frac{H\left(F^{\prime \prime}\right)^{2}}{\left(F^{\prime}\right)^{2}} \nu_{H}+\frac{H F^{\prime \prime \prime}}{F^{\prime}} \nu_{H}\right)+\frac{2 F^{\prime} c}{H^{4}}\right], \\
Q_{1}^{*}(H, \theta)=\frac{1}{H}\left[\partial\left(\frac{\cos \theta}{H} F^{\prime}, w^{*}\right) / \partial(H, \theta)\right], \\
Q_{2}^{*}(H, \theta)=\frac{1}{H}\left[\partial\left(-\frac{\sin \theta}{H} F^{\prime}, w^{*}\right) / \partial(H, \theta)\right],
\end{gathered}
$$

as calculated from substitution of (52) into (32) to (36).

One solution of (53), (54) such that $F^{\prime}(\theta) \neq 0$ is

$$
F(\theta)=N_{1} \theta+N_{2}, \quad \alpha^{*}(H, \theta)=\cot ^{-1}\left(N_{3} H^{2}\right)
$$

where $N_{1} \neq 0, N_{2}, N_{3}$ are arbitrary constants. Therefore

$$
\begin{aligned}
& L^{*}(H, \theta)=N_{1} \theta+N_{2} \text { i.e. } L\left(H_{1}, H_{2}\right)=N_{1} \tan ^{-1}\left(\frac{H_{2}}{H_{1}}\right)+N_{2}, \\
& \alpha^{*}(H, \theta)=\cot ^{-1}\left(N_{3} H^{2}\right) \text { i.e. } \alpha\left(H_{1}, H_{2}\right)=\cot ^{-1}\left(N_{3} H_{1}^{2}+N_{3} H_{2}^{2}\right)
\end{aligned}
$$

is a solution set of the partial differential equations (30), (31) or (20), (26).

As $N_{3}$ is arbitrary we can consider two separate cases of the solution (56). First, if $N_{3} \neq 0$, the flows will be variably inclined and second, if $N_{3}=0$, the flows will be crossed. For the case of $N_{3} \neq 0$, the functions $L^{*}(H, \theta)$ and $\alpha^{*}(H, \theta)$ are given by (56). 
Using (56) the flow variables in the physical plane are found to be

$$
\begin{aligned}
& H_{1}(x, y)=\frac{N_{1} x}{x^{2}+y^{2}}, \quad H_{2}(x, y)=\frac{N_{1} y}{x^{2}+y^{2}}, \\
& \left.u(x, y)=\frac{c}{N_{1}}\left(y+\frac{N_{3} N_{1}^{2} x}{x^{2}+y^{2}}\right), \quad v(x, y)=\frac{c}{N_{1}}\left(\frac{N_{3} N_{1}^{2} y}{x^{2}+y^{2}}\right)-x\right), \\
& w(x, y)=-\frac{2 c}{N_{1}}, j(x, y)=0, \quad \alpha(x, y)=\cot ^{-1}\left(\frac{N_{1}^{2} N_{3}}{\left(x^{2}+y^{2}\right)}\right), \\
& p(x, y)=\frac{\rho c^{2}}{2 N_{1}^{2}}\left(x^{2}+y^{2}\right)+2 \rho c^{2} N_{3} \tan ^{-1}\left(\frac{y}{x}\right)-\frac{\rho c^{2} N_{3}^{2} N_{1}^{2}}{2\left(x^{2}+y^{2}\right)}+N_{4},
\end{aligned}
$$

where $N_{4}$ is an arbitrary constant.

Using (57) the magnetic lines and streamlines are found to be

$$
\begin{gathered}
y / x=\text { const. }, \\
\left(x^{2}+y^{2}\right)+2 N_{3} N_{1}^{2} \tan ^{-1}(y / x)=\text { const. }
\end{gathered}
$$

respectively. Therefore, the result of this case is

THEOREM III. If the flow is a general MHD steady plane flow, then $L^{*}(H, \theta)=M_{1} H^{2}+$ $M_{2}$ is a Legendre transform function of a magnetic flux function such that the magnetic lines and the streamlines corresponding to this Legendre transform function and the variable angle $\alpha(x, y)=\cot ^{-1}\left(N_{1}^{2} N_{3} /\left(x^{2}+y^{2}\right)\right)$ are given by (58).

The solutions for the other case is easily obtained by taking $N_{3}=0$ in (57).

Application 3. Let

$$
L^{*}(H, \theta)=A \theta+B \ln H+D
$$

in $(H, \theta)$-coordinates where $A, B$ are non-zero real numbers and $D$ is an arbitrary constant.

Employing (59) in (30), the following partial differential equation is obtained

$$
B \frac{\partial \cot \alpha^{*}}{\partial \theta}-A H \frac{\partial \cot \alpha^{*}}{\partial H}+2 A \cot \alpha^{*}+2 B=0 .
$$

The general solution of this equation is

$$
\cot \alpha^{*}=-\frac{B}{A}+H^{2} F\left(\theta+\frac{B}{A} \ln H\right)
$$

where $F(\theta+B / A \ln H)$ is an arbitrary function of its argument.

Using (59), (61) in (32) to (36) we find,

$$
\begin{aligned}
& J^{*}=-\frac{H^{4}}{A^{2}+B^{2}}, \quad j^{*}=0, \quad w^{*}=-\frac{H^{2}}{A} c\left[F^{\prime}+\frac{2}{H^{2}}\right] \\
& Q_{1}^{*}=\frac{c}{H}\left[\frac{\left(B^{2}+A^{2}\right)}{A^{2}} \cos \theta F^{\prime \prime}+\frac{2 B}{A} \cos \theta F^{\prime}-2 \sin \theta F^{\prime}\right] \\
& Q_{2}^{*}=-\frac{c}{H}\left[\frac{\left(B^{2}+A^{2}\right)}{A^{2}} \sin \theta F^{\prime \prime}+\frac{2 B}{A} \sin \theta F^{\prime}+2 \cos \theta F^{\prime}\right] .
\end{aligned}
$$


From substituting (62) in (31) a second partial differential equation results,

$$
\frac{\eta H c}{A^{2}}\left[F^{\prime \prime \prime} \frac{\left(A^{2}+B^{2}\right)}{A}+4 B F^{\prime \prime}+4 A F^{\prime}\right]+\frac{\rho c^{2}}{H}\left[F^{\prime \prime} \frac{\left(A^{2}+B^{2}\right)}{A^{2}}+2 H^{2} F^{\prime} F\right]=0
$$

One solution of (63) is

$$
F\left(\theta+\frac{B}{A} \ln H\right)=M_{1}
$$

where $M_{1}$ is an arbitrary constant. Therefore

$$
\begin{aligned}
& \cot \alpha^{*}=-\frac{B}{A}+M_{1} H^{2}, \\
& L^{*}(H, \theta)=A \theta+B \ln H+D
\end{aligned}
$$

forms a solution set of the partial differential equations (30), (31).

Here $M_{1}$ is an arbitrary constant and thus two separate cases of the solution (64) may be considered. If $M_{1} \neq 0$, the flows are variably inclined. If $M_{1}=0$, the flows are constantly inclined with $\alpha=\cot ^{-1}(-B / A)$. When $M_{1} \neq 0$, the functions $L^{*}(H, \theta)$ and $\alpha^{*}(H, \theta)$ are given by (64).

Using (64), as in previous examples, the flow variables in the physical plane are found. They are as follows,

$$
\begin{aligned}
& H_{1}(x, y)=\frac{A x-B y}{x^{2}+y^{2}}, \quad H_{2}(x, y)=\frac{B x+A y}{x^{2}+y^{2}}, \\
& w(x, y)=-\frac{2 c}{A}, j(x, y)=0, \quad J(x, y)=-\frac{\left(A^{2}+B^{2}\right)}{\left(x^{2}+y^{2}\right)^{2}}, \\
& u(x, y)=c\left[\frac{y}{A}+\frac{M_{1}(A x-B y)}{x^{2}+y^{2}}\right], \\
& v(x, y)=c\left[-\frac{x}{A}+\frac{M_{1}(B x+A y)}{x^{2}+y^{2}}\right] \text {, } \\
& \alpha(x, y)=\cot ^{-1}\left(-\frac{B}{A}+\frac{M_{1}\left(A^{2}+B^{2}\right)}{x^{2}+y^{2}}\right) \text {, } \\
& \rho(x, y)=\frac{c^{2} \rho}{2 A^{2}}\left(x^{2}+y^{2}\right)-\frac{B}{A} c^{2} \rho M_{1} \ln \left(x^{2}+y^{2}\right)+2 c^{2} \rho M_{1} \tan ^{-1}\left(\frac{y}{x}\right) \\
& -c^{2} \frac{M_{1}^{2} \rho}{2} \frac{\left(A^{2}+B^{2}\right)}{\left(x^{2}+y^{2}\right)}+\frac{M_{1} B}{A} \rho c^{2}+M_{2}
\end{aligned}
$$

where $M_{2}$ is an arbitrary constant.

The magnetic lines are

$$
\frac{B}{2} \ln \left(x^{2}+y^{2}\right)-A \tan ^{-1}\left(\frac{y}{x}\right)=\text { const. }
$$


and the streamlines are

$$
\left(x^{2}+y^{2}\right)+2 M_{1} A^{2} \tan ^{-1}\left(\frac{y}{x}\right)-M_{1} A B \ln \left(x^{2}+y^{2}\right)=\text { const. }
$$

Summing up, we have

TheOREM IV. If the flow is a general MHD steady plane flow, then $L^{*}(H, \theta)=A \theta+$ $B \ln H+D$ is a Legendre transform function of a magnetic flux function such that the magnetic lines and streamlines corresponding to this Legendre transform function and the variable angle

$$
\alpha(x, y)=\cot ^{-1}\left(-\frac{B}{A}+\frac{M_{1}\left(A^{2}+B^{2}\right)}{x^{2}+y^{2}}\right)
$$

are given by (66) and (67) respectively.

By taking $M_{1}=0$ in (65) the solutions for the case constantly inclined flow can be obtained.

\section{REFERENCES}

[1] H. Alfven, On the motion of a charged particle in a magnetic field, Ark. Mat. Astr. Fys. 27A, 20 (1940)

[2] J. G. Kingston and R. F. Talbot, The solutions to a class of magnetohydrodynamic flows with orthogonal magnetic and velocity field distributions, Z. Angew. Math. Phys. 20, 956-965 (1969)

[3] J. S. Waterhouse and J. G. Kingston, Plane magneto hydrodynamic flows with constantly inclined magnetic and velocity fields, Z. Angew. Math. Phys. 24, 653-658 (1973)

[4] O. P. Chandna and V. I. Nath, On the uniqueness of MHD aligned flows with given streamlines, Canad. J. Phys. 5, 661-665 (1972)

[5] V. I. Nath and O. P. Chandna, On plane viscous magnetohydrodynamic flows, Quart. of Appl. Math. 31, $351-362(1973)$

[6] V. I. Nath and O. P. Chandna, On plane viscous MHD flows: II, Quart. of Appl. Math. 32, 337-341 (1974)

[7] O. P. Chandna and G. P. Mathur, Steady viscous magnetohydrodynamic flow in two dimensions with orthogonal magnetic and velocity field distributions, Tensor, N.S. 28, 269-272 (1974)

[8] O. P. Chandna, H. Toews and V. I. Nath, Plane MHD steady flows with constantly inclined magnetic and velocity fields, Canad. J. Phys. 53, 2613-2616 (1975)

[9] M. R. Garg and O. P. Chandna, Viscous orthogonal MHD flows, SIAM J. Appl. Math. 30, 577-585 (1976)

[10] O. P. Chandna and M. R. Garg, The flow of a viscous MHD fluid, Quart of Appl. Math. 34, 287-299 (1976)

[11] O. P. Chandna and H. Toews, Plane constantly-inclined MHD flow with isometric geometry, Quart. of Appl. Math. 34, 331-337

[12] O. P. Chandna and M. R. Garg, On steady plane magnetohydrodynamic flows with orthogonal magnetic and velocity fields, Int. J. Engng. Sci. 17, 251-257 (1979)

[13] R. M. Barron, O. P. Chandna and M. R. Garg, Solutions in viscous constantly-inclined magnetohydrodynamics, Jap. J. of Appl. Phys. 20, 167-173 (1981)

[14] R. M. Barron and O. P. Chandna, Hodograph transformations and solutions in constantly-inclined MHD plane flows, J. Engng. Math. 15, 211-220 (1981)

[15] O. P. Chandna and R. M. Barron, Plane constantly inclined magnetogasdynamic flows, Int. J. Engng. Sci. 19, 409-419 (1981)

[16] W. F. Ames, Nonlinear partial differential equations in engineering, Academic Press, New York, 1965

[ 17] P. Smith, Substitution principle for MHD flows, J. Math. Mech. 12, 505-520 (1963)

[18] S. I. Pai, Magnetogasdynamics and plasma dynamics, Springer-Verlag, Vienna, 1962

[19] M. H. Martin, The flow of a viscous fluid I, Arch. Rat. Mech. Anal. 41, 266-286 (1971) 